in vivo $35: 1545-1548(2021)$

doi:10.21873/invivo.12408

\title{
Analyzing the Expression of Biomarkers in Prostate Cancer Cell Lines
}

\author{
CHEN-YING SU ${ }^{1 \#}$, GWO-CHE HUANG ${ }^{2 \#}$, YOU-CHENG CHANG ${ }^{1}$, YU-JEN CHEN ${ }^{2}$ and HSU-WEI FANG ${ }^{1,3}$ \\ ${ }^{1}$ Department of Chemical Engineering and Biotechnology, \\ National Taipei University of Technology, Taipei, Taiwan, R.O.C.; \\ ${ }^{2}$ Department of Radiation Oncology, MacKay Memorial Hospital, Taipei, Taiwan, R.O.C.; \\ ${ }^{3}$ Institute of Biomedical Engineering and Nanomedicine, \\ National Health Research Institute, Zhunan, Taiwan, R.O.C.
}

\begin{abstract}
Background/Aim: CD44 and CD133 have been implicated as biomarkers of cancer cells and their expression could be analyzed to identify circulating tumor cells. Although CD44 and CD133 have been shown to be expressed in prostate cancer cells, a differential expression pattern has been reported depending on the tumor stage and cell line examined. We further investigated CD44 and CD133 expression in different prostate cancer cell lines to confirm whether their expression is distinguishable among patients with various tumor stages. Materials and Methods: CWR22Rv1, PC3, LNCaP, and DU145 cell lines were cultured and the cell morphology was observed for three days. The single expression of CD44 or CD133 and their combined expression were analyzed by flow cytometry. Results: We report that the single expression of CD133 was less than 5\% in all cell lines examined here. PC3 and DU145 cells displayed a high expression of CD44 (>93\%), while the expression of CD44 was less than $4 \%$ in CWR22Rv1 and LNCaP cells. CWR22Rv1 was the only cell line that demonstrated a high co-expression of both CD44 and CD133. Conclusion: Both single and combined expression of CD44 and CD133 should be considered when validating the detection of prostate cancer cells in circulating tumor cells.
\end{abstract}

This article is freely accessible online.

\#These Authors equally contributed to this work.

Correspondence to: Dr. Hsu-Wei Fang, Department of Chemical Engineering and Biotechnology, National Taipei University of Technology,1, Sec. 3, Zhongxiao E. Rd., Taipei 10608, Taiwan, R.O.C. Tel: +886 27712171 ext 2521, Fax: +886 27418575, email: hwfang@ntut.edu.tw

Key Words: Biomarker, prostate cancer, cancer stem cells, circulating tumor cells, CD44, CD133.
In developed countries, prostate cancer has become a leading cause of death among men (1). Men aged over 55 are recommended to have prostate-specific antigen screening (2, $3)$. Among all the screening methods, circulating tumor cell (CTC) detection is currently an accepted method for early cancer detection and tumor progression monitoring (4). Therefore, the selection of biomarkers is critical in order to assure the accuracy of CTC detection results.

$\mathrm{CD} 44^{+} / \alpha 2 \beta 1^{\text {high }} / \mathrm{CD} 133^{+}$cells have been isolated from DU145 prostate cancer cells as putative cancer stem cells (5). The combination of $\mathrm{CD} 44^{+} \mathrm{CD} 133^{+}$expression was enriched in PC3 and DU145 prostate cancer cells, and these CD $44^{+} \mathrm{CD} 133^{+}$ cells had a tumor-initiating potential (6). In addition, $\mathrm{CD} 44^{+} \mathrm{CD} 24^{-}$cells displayed stem cell properties when isolated from LNCaP cells (7). Most studies have focused only on the expression of individual or combined biomarkers without taking into account the expression level, which may provide false positive or false negative results while using CTCs for detecting prostate cancer. Therefore, we investigated the expression of both CD44 and CD133 in various human prostate cancer cell lines to evaluate whether they can be reliable biomarkers for prostate cancer detection in CTCs.

\section{Materials and Methods}

Cell lines and cell culture. The CWR22Rv1, PC3, LNCaP and DU145 prostate cancer cell lines were purchased from the American Type Culture Collection (ATCC, Manassas, VA, USA). Cells were cultured in RPMI 1640 (Roswell Park Memorial Institute 1640, GIBCO, Thermo Fisher Scientific, Waltham, MA, USA), containing 10\% FBS (Fetal bovine serum, Thermo Fisher Scientific), 1\% sodium pyruvate (Hyclone, GE Healthcare, Pittsburgh, PA, USA), $1.5 \mathrm{~g} / 1$ sodium biscarbonate (Sigma, St. Louis, MO, USA), and $1 \%$ penicillin/streptomycin (GIBCO, Thermo Fisher Scientific). The initial seeding concentration was $1 \times 10^{5}$ cells $/ \mathrm{ml}$ and cells were cultured at $37^{\circ} \mathrm{C}$ in a $5 \% \mathrm{CO}_{2}$ incubator. Cell growth was observed at 24 (Day 1), 48 (Day 2), and 72 hours (Day 3) after seeding. 

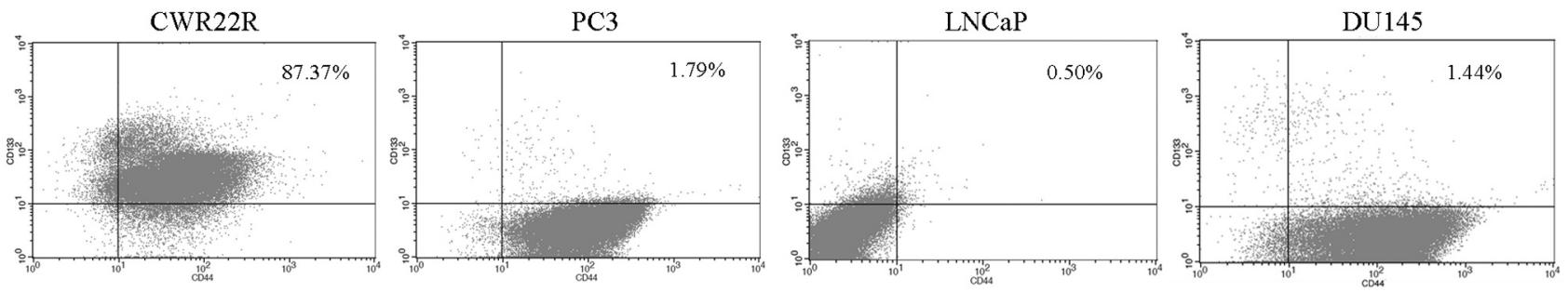

Figure 1. Expression of CD44 and CD133, and their co-expression in CWR22Rv1, PC3, LNCaP and DU145 cells, analysed by flow cytometry. The co-expression of CD44 and CD133 is denoted as percentage of total cells in the right top quarter.

Cell concentration by cell count. Cells were trypsinized after being cultured for 3 days and were concentrated by centrifugation. Cells were resuspended in $1 \mathrm{ml}$ of phosphate buffered saline (PBS, UniRegion Bio-Tech, Taiwan, ROC), and $100 \mu$ l of cells was transferred into a new microcentrifuge tube. An equal volume of of trypan blue was then added and $20 \mu \mathrm{l}$ of stained cells were loaded onto a hemocytometer and counted.

Flow cytometric analysis. CD44 (BD Pharmingen, San Diego, CA, USA) and CD133 (BioLegend, San Diego, CA, USA) were analyzed in this study $(8,9)$. Prostate cancer stem cells were washed with phosphate buffered saline (PBS, UniRegion Bio-Tech), and stained with antibodies for 30 minutes on ice in the dark. The samples were then washed with PBS for 3 times, and analyzed by the FACScalibur flow cytometer (Becton Dickinson, Franklin Lakes, NJ, USA). The flow cytometry results were evaluated using CellQuest Pro (BD Biosciences, Franklin Lakes, NJ, USA). The flow cytometry experients were repeated three times for each cell line, and 5,000 cells were analyzed each time. The expression pattern in the Figure 1 dotplots was from one representative flow cytometry experiment.

\section{Results}

The initial seeding concentration of all the prostate cancer cell lines was the same, and the morphology of cells was round at day 1 (Figure 2A). LNCaP cells were more elongated, and the other three cell lines were flatter at day 3 (Figure 2C). The density of LNCaP cells was lower after 1 day, while the density of CWR22Rv1 and PC3 was similar. Indeed, we observed the density of CWR22Rv1, PC3, and DU145 was higher than the density of LNCaP cells at day 3 (Table I).

Despite the differences in the proliferation rates, all cells were cultured to achieve the confluence for flow cytometric analysis. The expression of CD133 was low in all cell lines, while the expression of CD44 was distinct (Table II). The expression of CD44 was over 93\% in PC3 and DU145 cells, and less than $4 \%$ in CWR22Rv1 and LNCaP cells (Table II). The co-expression of CD44 and CD133 was around 87\% in CWR22Rv1 cells, but the co-expression was lower than $3 \%$ in PC3, LNCaP, and DU145 cells (Table II and Figure 1).
Table I. Cell concentration of different prostate cancer cell lines after culturing for 3 days. The initial concentration used for seeding was $1 \times 10^{5} \mathrm{cells} / \mathrm{ml}$

\begin{tabular}{lcccc}
\hline Cancer cell line & CWR22Rv1 & PC3 & LNCaP & DU145 \\
\hline Cells $/ \mathrm{ml}$ & $1.3 \times 10^{7}$ & $0.92 \times 10^{7}$ & $0.08 \times 10^{7}$ & $1 \times 10^{7}$ \\
\hline
\end{tabular}

Table II. The single and combined expression of CD44 and CD133 in different prostate cancer cell lines. The numbers represent mean \pm standard deviation.

\begin{tabular}{lcccc}
\hline Marker & CWR22Rv1 & PC3 & LNCaP & DU145 \\
\hline CD44+ $\left.^{+} \%\right)$ & $3.17 \pm 3.69$ & $93.35 \pm 3.53$ & $0.16 \pm 0.13$ & $96.92 \pm 0.93$ \\
$\mathrm{CD} 133^{+}(\%)$ & $2.38 \pm 2.43$ & $1.74 \pm 2.92$ & $4.71 \pm 0.49$ & $0.18 \pm 0.16$ \\
$\mathrm{CD}^{+} 4^{+} \mathrm{CD} 133^{+}(\%)$ & $87.04 \pm 1.99$ & $2.32 \pm 2.57$ & $0.33 \pm 0.28$ & $0.72 \pm 0.64$ \\
\hline
\end{tabular}

\section{Discussion}

Prostate cancer cell lines used in this study were derived from patients with different conditions (10). Each cell line contains different mutations, resulting in distinct proliferation rates. The morphology of each cell line was similar to what has been previously reported (11). We observed a faster growth rate for CWR22Rv1, PC3, and DU145 cells, and a slower proliferation for LNCaP cells. Indeed, it has been shown that the doubling time of CWR22Rv1, PC3, and DU145 is between 33 and 40 hours while LNCaP is between 60 and 72 hours (10). The proliferation of $\mathrm{LNCaP}$ has been shown to be androgenresponsive, thus $\mathrm{LNCaP}$ cells needed to be cultured in androgen-reducing or serum free medium for a faster growth $(11,12)$.

Concerning CD133, our results showed that its expression was very low. It was not surprising since it has been shown 


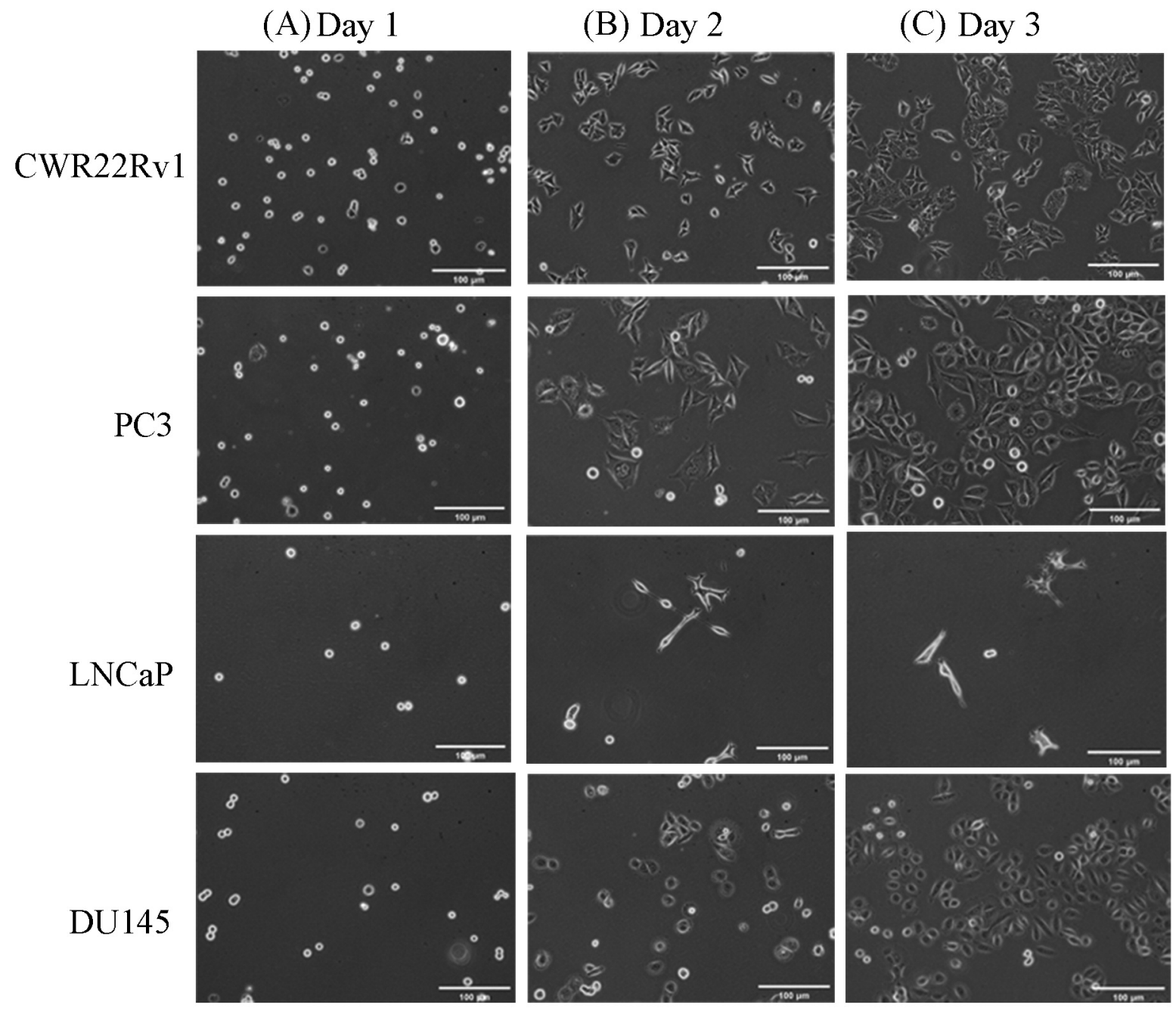

Figure 2. Morphology of CWR22Rv1, PC3, LNCaP and DU145 cells when cultured for 1, 2, and 3 days. The scale bar represents $100 \mu \mathrm{m}$.

that the expression of CD133 is extremely low in tumors isolated from prostate cancer patients, and the expression is even lower when cells are passaged multiple times (13). Similarly, when $\mathrm{CD}_{133^{+}}$cells were sorted from the CWR22Rv1 cell line, only $6 \%$ of cells remained CD133 ${ }^{+}$ after 2 weeks of culture (14). Therefore, it is still unclear whether CD133 is critical for the maintenance of prostate cancer cell characteristics.

Our results demonstrated that the high expression of CD44 was only observed in PC3 and DU145 cells. The single expression of CD44 was low in CWR22Rv1 cells, but the co-expression of CD44 and CD133 was high. It has been shown that CD44 is associated with prostate cancer proliferation (15). It is possible that the faster proliferation rate we observed in PC3, DU145, and CWR22Rv1 could correspond to the high expression of CD44 and/or CD133.
In addition, $\mathrm{CD}_{133^{+}}$cells have been demonstrated to proliferate faster than CD133- cells (16). The low expression of CD44 and CD133 in LNCaP cells might be associated with their slow proliferation rate. However, whether the expression of CD44 and CD133 affects the proliferation rate or vice versa requires further investigation.

We could divide prostate cancer stem cell lines into three groups: DU145 and PC3 were CD44 ${ }^{\text {high }} \mathrm{CD} 133^{\text {low }}$, CWR22Rv1 was $\left(\mathrm{CD} 44^{+} \mathrm{CD} 133^{+}\right)^{\text {high }}$, and LNCaP displayed CD $44^{\text {low }} \mathrm{CD} 133^{\text {low }}$ characteristics. We speculate that these results reflect the limit of detecting prostate cancer using CTCs when only considering the expression of biomarkers. In future studies, we will focus on characterizing the biomarkers' expression of prostate cancer cell lines under various differentiation stages to mimic the clinical situation of patients with different tumor stages. 


\section{Conclusion}

In this study, the distinct cell proliferation rates were demonstrated when four different prostate cancer cell lines were cultured. The distinct expression pattern of CD44 and CD133 in different prostate cancer cell lines was also shown, suggesting that both single and co-expression of CD44 and CD133 should be taken into account when identifying prostate cancer cells in CTCs in order to evaluate the state of prostate cancer progression.

\section{Conflicts of Interest}

The Authors declare no conflicts of interest.

\section{Authors' Contributions}

All Authors contributed to the study conception and design. G.-C Huang, and Y.-C. Chang performed laboratory experiments. C.-Y. Su, G.-C. Huang, and Y.-C. Chang analyzed data. C.-Y. Su wrote the manuscript. Y.-J. Chen and H.-W. Fang reviewed the data and analysis, and revised the manuscript. All Authors have read and approved the final manuscript.

\section{Acknowledgements}

This study was supported by the National Taipei University of Technology and Mackay Memorial Hospital Joint Research Program, NTUT-MMH-107-02.

\section{References}

1 Bell KJ, Del Mar C, Wright G, Dickinson J and Glasziou P: Prevalence of incidental prostate cancer: A systematic review of autopsy studies. Int J Cancer 137(7): 1749-1757, 2015. PMID: 25821151. DOI: $10.1002 /$ ijc. 29538

2 Ankerst DP, Gelfond J, Goros M, Herrera J, Strobl A, Thompson IM Jr, Hernandez J and Leach RJ: Serial percent free prostate specific antigen in combination with prostate specific antigen for population based early detection of prostate cancer. J Urol 196(2): 355-360, 2016. PMID: 26979652. DOI: 10.1016/j.juro. 2016.03.011

3 Carlsson S, Assel M, Ulmert D, Gerdtsson A, Hugosson J, Vickers A and Lilja H: Screening for prostate cancer starting at age 50-54 years. A population-based cohort study. Eur Urol 71(1): 46-52, 2017. PMID: 27084245. DOI: 10.1016/j.eururo.2016.03.026

4 Ried K, Eng P and Sali A: Screening for circulating tumour cells allows early detection of cancer and monitoring of treatment effectiveness: An observational study. Asian Pac J Cancer Prev 18(8): 2275-2285, 2017. PMID: 28843267. DOI: 10.22034/ APJCP.2017.18.8.2275

5 Wei C, Guomin W, Yujun L and Ruizhe Q: Cancer stem-like cells in human prostate carcinoma cells DU145: The seeds of the cell line? Cancer Biol Ther 6(5): 763-768, 2007. PMID: 17592251. DOI: 10.4161/cbt.6.5.3996

6 Dubrovska A, Kim S, Salamone RJ, Walker JR, Maira SM, García-Echeverría C, Schultz PG and Reddy VA: The role of
PTEN/Akt/PI3K signaling in the maintenance and viability of prostate cancer stem-like cell populations. Proc Natl Acad Sci USA 106(1): 268-273, 2009. PMID: 19116269. DOI: 10.1073/pnas.0810956106

7 Hurt EM, Kawasaki BT, Klarmann GJ, Thomas SB and Farrar WL: CD44+ CD24(-) prostate cells are early cancer progenitor/stem cells that provide a model for patients with poor prognosis. Br J Cancer 98(4): 756-765, 2008. PMID: 18268494. DOI: $10.1038 /$ sj.bjc. 6604242

8 Dubrovska A, Elliott J, Salamone RJ, Telegeev GD, Stakhovsky AE, Schepotin IB, Yan F, Wang Y, Bouchez LC, Kularatne SA, Watson J, Trussell C, Reddy VA, Cho CY and Schultz PG: CXCR4 expression in prostate cancer progenitor cells. PLoS One 7(2): e31226, 2012. PMID: 22359577. DOI: 10.1371/ journal.pone. 0031226

9 Wang L, Huang X, Zheng X, Wang X, Li S, Zhang L, Yang Z and Xia Z: Enrichment of prostate cancer stem-like cells from human prostate cancer cell lines by culture in serum-free medium and chemoradiotherapy. Int J Biol Sci 9(5): 472-479, 2013. PMID: 23781140. DOI: 10.7150/ijbs.5855

10 Cunningham D and You Z: In vitro and in vivo model systems used in prostate cancer research. J Biol Methods 2(1):e17, 2015. PMID: 26146646. DOI: 10.14440/jbm.2015.63

11 Marchiani S, Tamburrino L, Nesi G, Paglierani M, Gelmini S, Orlando C, Maggi M, Forti G and Baldi E: Androgen-responsive and -unresponsive prostate cancer cell lines respond differently to stimuli inducing neuroendocrine differentiation. Int $\mathrm{J}$ Androl 33(6): 784-793, 2010. PMID: 20088946. DOI: 10.1111/j.13652605.2009.01030.x

12 Horoszewicz JS, Leong SS, Kawinski E, Karr JP, Rosenthal H, Chu TM, Mirand EA and Murphy GP: LNCaP model of human prostatic carcinoma. Cancer Res 43(4): 1809-1818, 1983. PMID: 6831420 .

13 Collins AT, Berry PA, Hyde C, Stower MJ and Maitland NJ: Prospective identification of tumorigenic prostate cancer stem cells. Cancer Res 65(23): 10946-10951, 2005. PMID: 16322242. DOI: 10.1158/0008-5472.CAN-05-2018

14 Vander Griend DJ, Karthaus WL, Dalrymple S, Meeker A, DeMarzo AM and Isaacs JT: The role of CD133 in normal human prostate stem cells and malignant cancer-initiating cells. Cancer Res 68(23): 9703-9711, 2008. PMID: 19047148. DOI: 10.1158/0008-5472.CAN-08-3084

15 Ni J, Cozzi PJ, Hao JL, Beretov J, Chang L, Duan W, Shigdar S, Delprado WJ, Graham PH, Bucci J, Kearsley JH and Li Y: CD44 variant 6 is associated with prostate cancer metastasis and chemo-/radioresistance. Prostate 74(6): 602-617, 2014. PMID: 24615685. DOI: $10.1002 / \operatorname{pros} .22775$

16 Kelly SE, Di Benedetto A, Greco A, Howard CM, Sollars VE, Primerano DA, Valluri JV and Claudio PP: Rapid selection and proliferation of $\mathrm{CD} 133+$ cells from cancer cell lines: Chemotherapeutic implications. PLoS One 5(4): e10035, 2010. PMID: 20386701. DOI: 10.1371/journal.pone.0010035

Received March 10, 2021

Revised March 25, 2021

Accepted March 30, 2021 